\title{
ANÁLISE DO COMPORTAMENTO E O ESTUDO DO ENVELHECIMENTO HUMANO: REVISÃO DOS ESTUDOS DE APLICAÇÃO
}

\author{
BEHAVIOR ANALYSIS AND THE STUDY OF HUMAN AGING: \\ A REVIEW OF APPLIED STUDIES
}

\author{
Celso Goyos ${ }^{1}$ \\ UNIVERSIDADE FEDERAL DE SÃO CARLOS
}

Rosana Ap. Salvador Rossit ${ }^{2}$

UNIVERSIDADE FEDERAL DE SÃO PAULO

\author{
Nassim Chamel Elias ${ }^{3}$, Giovana Escobal ${ }^{4}$ e Paulo Chereguini $^{5}$ \\ UNIVERSIDADE FEDERAL DE SÃO CARLOS
}

\begin{abstract}
RESUMO
O ensino de habilidades e o uso de procedimentos comportamentais têm demonstrado efetividade no cuidado de idosos dependentes, aumentando e/ou mantendo habilidades funcionais e evitando a institucionalização. O presente artigo objetivou levantar dados na literatura da área que deem suporte para o uso de procedimentos e técnicas em análise do comportamento, que possam ser eficazes no tratamento de disfunções comportamentais em população idosa. Realizou-se um levantamento bibliográfico em diversos periódicos da área de análise do comportamento, destacando-se os artigos que tinham como alvo populações idosas, seus cuidadores e instituições que prestam serviços a essa clientela. Foram analisados 16 artigos. Esses artigos apresentam estudos sobre memória, comportamento de vaguear, redução da incontinência urinária e fala desconexa. Os resultados sugerem eficácia no uso de intervenções comportamentais com idosos e a necessidade de estudos adicionais utilizando delineamentos experimentais mais rigorosos e medidas de validade social. Na atenção ao idoso, deve-se considerar que a manutenção, aquisição ou recuperação de uma habilidade traz benefícios pessoais, como autonomia, aumento da autoestima e melhor domínio de seu ambiente físico e social.
\end{abstract}

Palavras-chave: gerontologia, análise do comportamento, habilidades funcionais, independência do indivíduo idoso.

\begin{abstract}
Teaching skills and applied behavioral-based procedures have yielded effective care of dependent aging people, and improving and/or maintaining functional skills and avoiding institutionalization. The present paper consists of a literature review of behavior analytic procedures applied to the treatment of behavioral disorders in the aging population. Behavior Analysis journals were searched, focusing the articles that had aging participants as their target population, their caregivers and also institutions that provide services to the aging population. 16 articles were found and analyzed. These articles presented research on memory, wandering behavior, reduction of urinary incontinence and control of disruptive speech. The results highlighted behavioral procedures as effective forms of treatment of the aging population and the need for further studies using more rigorous experimental designs and measures of social validity. As far as caring for the aging population goes, it should be considered that acquisition, maintenance, or recovering skills brings about personal benefits, such as: greater autonomy, increase of self-esteem, better command of their physical and social environment.
\end{abstract}

Keywords: gerontology, behavior analysis, functional skills, aging people independence.

\footnotetext{
${ }^{1}$ Bolsista produtividade, CNPq Universidade Federal de São Carlos, Departamento de Psicologia. C.P. 676, Tel.: (16) 3351-8498, São Carlos/ SP, CEP: 13565-905 UFSCar. E-mail: celsogoyos@hotmail.com

${ }^{2}$ Universidade Federal de São Paulo, Campus Baixada Santista, Curso de Terapia Ocupacional, Santos/SP.

${ }^{3}$ Bolsista de pós-doutorado Fapesp, pesquisador associado do Departamento de Psicologia da UFSCar.

${ }^{4}$ Bolsista de doutorado Fapesp, Programa de Pós-graduação em Educação Especial da UFSCar.

${ }^{5}$ Mestre em Educação Especial, Programa de Pós-graduação em Educação Especial da UFSCar.
} 
A gerontologia é o campo que visa a descrever e explicar as mudanças típicas do processo de envelhecimento e de seus determinantes genético-biológicos, psicológicos e socioculturais. Abrange aspectos do envelhecimento normal e patológico. Compreende a identificação dos níveis atuais de desenvolvimento e do potencial para o desenvolvimento. A gerontologia comporta interfaces com outras áreas profissionais, entre as quais se destacam a clínica médica, a psiquiatria, a geriatria, a fisioterapia, a enfermagem, a terapia ocupacional, a psicologia, o serviço social e o direito. Dessas interfaces derivam soluções para problemas individuais e sociais, novas tecnologias, evidências e questões relevantes para investigação (Néri, 2001).

Estudos e pesquisas na área de gerontologia buscam soluções para tentar devolver ou restaurar habilidades e competências que os indivíduos perderam ao longo do curso de vida, como a interação social, a autonomia, a independência nas atividades do cotidiano, a memória, a comunicação e a valorização pela vida. É preciso entender que o idoso tem potencial para desempenhar diversas atividades e para continuar aprendendo quando se utilizam procedimentos de intervenção cuidadosamente delineados (Burgio \& Burgio, 1986).

Essa questão se torna ainda mais relevante devido ao aumento da expectativa de vida dos seres humanos. Com o envelhecimento populacional, surge a necessidade de transformações socioeconômicas e educacionais, visando à melhoria da qualidade de vida dos idosos e daqueles que estão envelhecendo (Carvalho Filho, 2000).

Uma das consequências das descobertas científicas e do desenvolvimento tecnológico é a longevidade humana. O Brasil já vivencia o aumento da expectativa de vida, que, em 2000, indicava 70,4 anos, e deverá atingir os 81,3 anos em 2050. Com efeito, a população brasileira maior de 60 anos passou de 4\% em 1940 para 9\% em 2000, gerando uma população de 15 milhões de idosos no país (Maria, 2003). Entretanto, de forma paradoxal, o tratamento social e familiar parece não proporcionar aos idosos uma vivência digna e de qualidade. Embora a legislação brasileira defenda os direitos de idosos ativos e participativos na comunidade, e o Estatuto do Idoso (Brasil, 2003) preze pelos direitos e exercício da cidadania, o retrato que se vê da situação dos idosos no Brasil é desalentador. Maria (2003) afirma que mais de 80\% dos idosos apresentam ao menos uma doença crônica e quase metade necessita de alguma ajuda na realização de ao menos uma das atividades diárias, e quase 10\% apresentam algum tipo de dependência, impedindo que residam sozinhos. Em geral, como consequência, a família ou instituições especializadas assumem o cuidado do idoso debilitado como forma de compensar a carência de recursos e de políticas de saúde adequadas para a assistência e o cuidado da população idosa (Creutzberg \& Santos, 2000). Observa-se, com frequência, o seu isolamento social, juntamente com os problemas de saúde advindos da existência frequente de múltiplas afecções concomitantes.

Dos problemas enfrentados pela população idosa, um dos mais frequentemente observados é o seu baixo grau de engajamento em atividades da vida diária e instrumental de vida prática, tais como deslocar-se fisicamente, alimentar-se, vestir-se e cuidar de sua higiene pessoal e ambiental. A ociosidade, a falta de perspectivas e a ausência ou despre- 
paro de pessoas especializadas para atender a essa clientela agravam ainda mais as dificuldades dessa população. Esse perfil pessoal e social é visto como algo imutável, sem perspectivas de melhora, e comumente tratado pela equipe multidisciplinar como um fato irreversível (Engelman, Altus, \& Mathews, 1999).

De acordo com Birren e Schaie (1996), nas últimas duas décadas as pesquisas têm indicado que as alterações anatômicas, funcionais e cognitivas decorrentes do processo de envelhecimento não são iguais para as diferentes faixas etárias; adicionalmente, apenas a idade cronológica usada como variável independente não ajuda a explicar o processo de envelhecimento. A questão central da pesquisa em envelhecimento diz respeito à relação entre idade como a variável independente primária e alguns outros fatores de interesse, como mortalidade, morbidade, autonomia, qualidade de vida, cognição e produtividade. As investigações também avaliam a extensão na qual algumas variáveis intervenientes, como fatores sociodemográficos, ambientais, psicossociais, biopsicológicos ou de estilo de vida, estão associadas às alterações observadas com a idade.

Historicamente, a gerontologia tem sido guiada por paradigmas sociológicos e de desenvolvimento, que levam em conta o fator biológico do envelhecimento como uma variável causal dos deficits e do desempenho das pessoas idosas. Sob outra perspectiva, a análise do comportamento propõe que a interação entre o organismo envelhecido e o ambiente em que ele se insere pode ser manipulada de forma a melhorar a qualidade de vida e a efetividade dos comportamentos emitidos pelos idosos (Birren \& Schaie, 1996; Burgio \& Burgio, 1986).
Para aumentar a probabilidade de esses indivíduos se tornarem independentes, reduzir comportamentos inadequados e diminuir sua necessidade de supervisão integral, a literatura na área de gerontologia comportamental desenvolveu uma sólida metodologia para avaliar esses processos e desenvolver tecnologias efetivas. Burgio e Burgio (1986) conceituaram a gerontologia comportamental como o estudo de como eventos ambientais antecedentes e consequentes interagem com o organismo envelhecido para produzir o comportamento.

Segundo o paradigma comportamental (Lindsley, 1964; Skinner, 1983), a pessoa idosa tem deficits que limitam a extensão de estímulos discriminativos que podem controlar comportamentos em ambientes habituais, e as contingências de reforçamento para os idosos podem conduzir a comportamentos não efetivos. Por exemplo, muitos reforçadores tendem a ocorrer em esquemas com reforçadores mais espaçados. Além disso, a partir do momento em que os comportamentos em idosos tendem a ser mais frequentemente seguidos por dores e fadiga, reforçadores positivos tornam-se menos comuns e menos poderosos, e reforçadores negativos, mais frequentes. Wisocki (1991) indica duas implicações importantes do uso de intervenções comportamentais com idosos: (1) o método de análise é funcional, ou seja, condições biológicas e comportamentais se influenciam mutuamente, e, decorrente disso, (2) a idade cronológica não é vista como variável causal, mas correlacional e descritiva.

Tendo em vista o potencial de aplicabilidade prática e a necessidade de sistematização de procedimentos, o presente estudo 
teve por objetivo levantar dados na literatura da área de gerontologia comportamental que apresentem procedimentos e técnicas da análise do comportamento e que possam ser eficazes no tratamento de problemas comportamentais em população idosa.

\section{MÉTODO}

Foram analisados 16 artigos, selecionados dentre aqueles publicados de 1980 a 2006, período imediatamente anterior ao trabalho, em volumes dos periódicos Journal of Applied Behavior Analysis, Journal of the Experimental Analysis of Behavior, Journal of Clinical Geropsychology, considerados aqueles em que mais provavelmente estariam publicados os artigos de interesse, em separatas avulsas de outros periódicos e em livros, tais como Care of the elderly: A family approach (Pinkston \& Linsk, 1984), em que artigos relacionados foram localizados em função de estarem citados nos artigos anteriormente identificados. Os dados foram coletados em uma das bases de dados da Capes, Web of Science, e no site <www.behavior.org>, assim como no acervo da biblioteca setorial do Laboratório de Aprendizagem Humana, Multimídia Interativa e Ensino Individualizado da UFSCar.

Cada artigo foi classificado de acordo com os seguintes critérios: área de conhecimento (psicologia, terapia ocupacional, medicina e fisioterapia), comportamento alvo (deficits em comportamento verbal e social, comportamento de vaguear, memória e tempo de reação, incontinência urinária e engajamento em atividades) e população (idosos, cuidadores e instituições), e os procedimentos utilizados foram descritos.

\section{Cálculo de concordância}

A classificação dos artigos em cada uma das categorias estabelecidas foi realizada por pelo menos dois dos autores para posterior cálculo de concordância. O índice de concordância foi obtido da seguinte maneira: em cada categoria, uma concordância era registrada se as classificações fossem as mesmas; caso contrário, uma discordância era registrada. A fórmula utilizada foi: o número de concordância dividido pelo número de concordância mais o número de discordância multiplicado por 100 (Hall, 1974). O índice de concordância foi de $100 \%$ para todas as categorias.

\section{Resultados}

\section{Memória e tempo de reação}

Um dos problemas mais importantes enfrentados pelos idosos relaciona-se à memória. Baron e Surdy (1990) levantaram a hipótese de que deficits em testes de memória podem refletir a limitação causada pela idade e uma relutância em relatar um estímulo ou evento visto anteriormente, provavelmente por causa de uma história de consequências aversivas geradas por relatos falsos.

Para testar essa hipótese, Baron e Surdy (1990) propuseram um estudo baseado em resposta de reconhecimento. Quatro idosos (de 62 a 75 anos) e quatro jovens (de 18 a 26 anos) foram submetidos a um procedimento em que um estímulo, de uma série de estímulos, aparecia na tela a cada quatro segundos. Foram programadas duas teclas distintas, uma correspondente a "novo" e outra a "velho", para a resposta de reconhecimento. A cada estímulo apresentado, era solicitado que o participante pressionasse a tecla "novo" 
para estímulos que apareciam pela primeira vez em uma sequência, ou a tecla "velho" para estímulos que já haviam aparecido nessa sequência. Os pesquisadores usaram como dados as medidas de intervalo de separação (entre a primeira e a segunda aparição de um mesmo estímulo, havia um número variável de itens intervenientes: 0, 2, 4, 8, 16, 32, 64), tipo de estímulo (sequências alfanuméricas, palavras ou frases curtas) e matriz de recompensa (contingência de reforçamento).

Os resultados evidenciaram que a prática da tarefa aumenta a habilidade de reconhecimento, particularmente no caso dos idosos, e que o avanço na idade pode contribuir para a redução de sensibilidade às mudanças de contingências, mas isso não constitui causa direta dos deficits apresentados. Os autores defendem a ideia de que a idade tem um papel importante na plasticidade comportamental, lembrando que os idosos estão expostos a ambientes relativamente estáveis, o que dificulta que os comportamentos de indivíduos mais velhos sejam modificados com facilidade quando as contingências ambientais são alteradas.

Além das questões relacionadas diretamente com a memória, alguns autores (Baron \& Menich, 1985; Baron, Menich, \& Perone, 1983) sugerem que a velocidade de resposta, ou tempo de reação, está inversamente relacionada com a idade.

Para verificar essa afirmação, Baron et al. (1983) e Baron e Menich (1985) utilizaram tarefas de escolha de acordo com o modelo, aplicadas por meio de um computador, para investigar o tempo de reação. Uma tentativa iniciava quando o participante pressionava duas teclas específicas. Então, um estímulo modelo era apresentado no centro do mo- nitor por dois segundos; em seguida, eram apresentados dois estímulos comparações, um à direita e outro à esquerda. Soltar a tecla correspondente ao estímulo comparação correto era contado como uma resposta correta.

No estudo de Baron et al. (1983), participaram sete homens com idade de 18 a 23 anos e sete de 63 a 79 anos. As respostas corretas dependiam de uma mensagem mostrada na tela: "Problema: Mesmo", o reforçador era contingente com soltar a tecla correspondente ao estímulo comparação igual ao modelo; "Problema: Diferente", a contingência exigia soltar a chave correspondente ao estímulo comparação diferente. Uma mensagem na tela indicava se a resposta dada estava correta ou não.

No estudo de Baron e Menich (1985), participaram cinco homens de 18 a 23 anos e cinco de 65 a 73 anos. Os estímulos modelos poderiam ser formados por um padrão simples ou por dois ou três padrões arranjados em uma linha horizontal. O estímulo comparação correto deveria ser um dos padrões do estímulo modelo. O par de estímulos comparações poderia aparecer imediatamente após a apresentação do modelo ou após atrasos de cinco, 10 ou 15 segundos. Em algumas tentativas com atraso, um som era apresentado por dois segundos imediatamente antes da apresentação dos estímulos comparações.

Os resultados desses dois estudos indicaram que os efeitos do treino foram aproximadamente os mesmos para os participantes mais jovens e mais velhos, ou seja, o desempenho de jovens e de idosos diferiu ao início do estudo e permaneceu diferente ao final do estudo, na mesma proporção. Esses resultados refletem uma mudança fisiológica 
fundamental no sistema nervoso. Entretanto, os procedimentos desses estudos ilustram a possibilidade de usar contingências operantes para modificar a velocidade do responder de adultos mais velhos, que cresceu substancialmente entre o início e o fim dos estudos.

Além disso, no estudo de Baron e Menich (1985), os resultados indicaram que o responder foi mais lento para os idosos, nas discriminações com atraso, o que pode ser interpretado como perda de memória de curto prazo. Os resultados também indicaram que os desempenhos foram facilitados quando as discriminações com atraso eram sinalizadas. Isso sugere que pelo menos um pouco da perda pode se dever à apresentação repentina dos estímulos comparações. O responder também se tornou mais lento conforme o número de elementos do modelo aumentou.

Plaud, Gillund, e Ferraro (2000) desenvolveram um estudo para determinar o quanto os processos de envelhecimento afetam os parâmetros de aprendizagem, a habilidade para operar em seu ambiente, a sensibilidade a consequências e a estímulos que estabelecem ocasião para as consequências e para coletar dados normativos com idosos sem demência para futuras pesquisas comparativas. Participaram cinco mulheres e um homem, com idades de 62 a 74 anos, sem demência diagnosticada. Foi utilizado um delineamento de sujeito único e de grupos entre os sujeitos. As tarefas iniciavam com uma mensagem apresentada no computador. Os sujeitos eram instruídos a pressionar o botão F1 ao ver o círculo branco e a apertar o botão F12 ao ver a letra "A" vermelha. Cada estímulo permanecia no monitor por quatro segundos. As respostas à tecla F1 foram refor- çadas em um esquema de intervalo variável (VI) de 30 segundos; as respostas à tecla F12, em um esquema de intervalo variável de 60 segundos. Em esquemas de intervalo variável, a apresentação de um reforçador depende da passagem de um período variável de tempo e da emissão de uma única resposta: as respostas que ocorrem antes do final do intervalo não têm efeito (Catania, 1999). Os valores 30 segundos e 60 segundos são as médias de períodos variáveis de tempo. Por exemplo, 30 segundos pode ser a média de quatro períodos: 40, 20, 30, 10 e 50 segundos.

Os resultados indicaram diferenças significativas nos tempos de resposta em cinco dos seis participantes. Para três participantes, as respostas foram mais rápidas no esquema de VI 30s. Para dois participantes, as respostas foram mais rápidas no esquema de VI 60s. Não foi notada diferença significativa para o outro participante. $\mathrm{O}$ controle por estímulos discriminativos no procedimento utilizado praticamente eliminou respostas incorretas pelos idosos do estudo. Esses resultados são encorajadores para o uso de estratégias comportamentais na modificação de comportamento por meio de controle de estímulos em idosos.

Deficits em comportamento verbal e comportamento social

Alguns dos problemas relacionados com o comportamento verbal em idosos são as verbalizações bizarras ou desconexas com a realidade e decorrentes deficits de comunicação. Membros da comunidade tendem a punir as verbalizações inapropriadas, causando a redução das oportunidades para o idoso conversar e perguntar, até a provável extinção da fala. Segundo Pinkston e Linsk (1984), falas 
excessivas, paranoicas, com ecolalia e repetitivas são geralmente tratadas como desordens psiquiátricas. Baixas taxas de verbalização, pouca iniciação de diálogos e poucas respostas podem resultar em diagnóstico de depressão, problema neurológico ou psicose.

Alguns estudos (Green, Linsk, \& Pinkston, 1986; Henry \& Horne, 2000; Pinkston \& Linsk, 1984) foram conduzidos no sentido de modificar esses comportamentos, utilizando procedimentos comportamentais baseados em reforçamento ou atenção diferencial. Nesses procedimentos, respostas apropriadas são diferencialmente reforçadas. O reforçamento dependerá das propriedades da resposta, tais como intensidade, propriedades temporais, topográficas ou outras, incluindo os estímulos e os eventos na presença dos quais ela é emitida (Catania, 1999). Henry e Horne (2000) ensinaram comportamentos verbais de falante (imitação e nomeação) e de ouvinte (atender a um pedido) por meio de reforçamento diferencial das respostas-alvo a cinco idosos com diagnóstico de demência. Alguns comportamentos aumentaram, enquanto outros, para o mesmo participante, diminuíram. Apesar de os resultados não mostrarem a consistência esperada, os autores sugerem que novas propostas possam se beneficiar da compreensão do estabelecimento de repertórios eficientes de falante e de ouvinte nessa população específica.

Uma alternativa promissora que pode ser associada ao procedimento de reforçamento diferencial é o treinamento e o uso de familiares e cuidadores como agentes fornecedores do reforço. Muitas vezes, esses agentes reforçam comportamentos inapropriados, aumentando a sua ocorrência, ou punem comportamentos desejáveis, acarretando a sua extinção. Podemos supor que, após emitirem gritos desconexos, idosos com demência sejam amparados com algum tipo de atenção; se essa atenção for reforçadora, aumenta a probabilidade de esse comportamento se repetir e se fortalecer.

Pinkston e Linsk (1984) planejaram uma intervenção para avaliar a efetividade do reforçamento diferencial para tratar problemas de fala em idosos, com as esposas como agentes de mudança, e verificar a generalização dos efeitos ao longo do tempo. Participaram dois casais de idosos cujos maridos apresentavam problemas de comportamento verbal. As esposas foram treinadas, por meio de técnicas de role-playing, instruções e demonstrações, a utilizar corretamente consequências para verbalizações adequadas. Avaliações constantes dos comportamentos-alvo foram ilustradas em gráficos e ajudaram as esposas e os experimentadores a examinarem repetidamente os procedimentos.

No estudo de Green et al. (1986), o marido de um dos casais, Sr. Orr, tinha 67 anos e havia sofrido um derrame três meses antes do início da intervenção. O outro marido, Sr. Ford, tinha 63 anos e já tivera uma série de derrames. Os comportamentos problemáticos foram definidos como verbalizações de suspeita e acusação ("tem outro homem na casa”) e verbalizações degradantes ("você é uma prostituta"). As respostas verbais apropriadas foram definidas como respostas a perguntas de outras pessoas e que tinham conteúdo, duração e tempo adequados, incluindo respostas do tipo "sim/não" e respostas espontâneas sem dicas por parte dos outros, incluindo perguntas, afirmações ou ordens, e que tivessem obrigatoriamente conteúdo adequado. Para o Sr. Ford, após a interven- 
ção, a frequência de respostas inadequadas permaneceu a mesma; porém, a duração total dos episódios caiu significativamente. $\mathrm{O}$ follow-up revelou que as durações dos episódios de verbalização inadequada permaneceram baixas e que a frequência dos episódios havia diminuído. Os dados mostraram que, antes da intervenção, a Sra. Ford negava ou discutia cerca de 95\% das verbalizações do Sr. Ford. Durante a intervenção e o follow-up, os números mudaram para cerca de 14\% de consequências para verbalizações inadequadas e $82 \%$ para verbalizações adequadas. Para o Sr. Orr, durante a intervenção, houve um aumento de respostas adequadas. $\mathrm{O}$ número de verbalizações espontâneas aumentou durante a intervenção, a manutenção e o follow-up. Os resultados indicaram que os procedimentos de reforçamento diferencial contingente a verbalizações apropriadas mostram um grande potencial para uso com idosos, principalmente se aplicados por familiares. Assim, os dados permitem sugerir o uso de técnicas comportamentais para treinar cuidadores e familiares a modificar comportamentos problemáticos de idosos.

Os problemas de verbalizações, juntamente com perda de memória e demência, acarretam outros deficits comportamentais. Um exemplo disso é que muitos idosos, principalmente os residentes em instituições de longa permanência, vivenciam algum grau de isolamento social na comunidade (Praderas \& MacDonald, 1986).

O problema de isolamento social nesses indivíduos é composto por, e contribui para, problemas como a depressão, diminuição da autoestima e da satisfação pela vida. Segundo Praderas e MacDonald (1986), os fatores que contribuem para o isolamento social são a inabilidade fisica, que torna difícil aos idosos deixarem a instituição sem assistência, e a perda de outras funções significativas, que contribuem para a redução das possibilidades de contato e participação na comunidade. Uma dessas funções refere-se à conversação. A melhora das características das conversações pode resultar em uma percepção mais positiva pelos membros da comunidade, diminuindo o isolamento.

Praderas e MacDonald (1986) pretendiam demonstrar que características qualitativas de um tipo específico de habilidade de conversação poderiam ser modificadas em idosos isolados socialmente. Para tanto, foi utilizado um programa de treinamento de habilidades de conversação por telefone com quatro idosos isolados socialmente (idades de 66, 68, 85 e 87 anos), que apresentavam limitações física, emocional e cognitiva. Durante o procedimento, o idoso ficava de um lado de uma mesa e o pesquisador do outro, com uma cortina entre os dois, para que o idoso pudesse ouvir, mas não pudesse ver o pesquisador. Foi utilizada a técnica de role-play. O treino foi conduzido em passos sequenciais, de forma que cada um dos participantes foi treinado para atingir o critério em um componente da habilidade, depois para outro componente, e assim por diante. Os quatro componentes foram: a) expressar cortesias comuns; b) fazer autodiscurso positivo; c) fazer perguntas; e d) fazer interjeições e reconhecimentos. Os resultados mostraram efeitos positivos com os quatro sujeitos; em dois casos, mudanças foram significativas o suficiente para afetar a percepção de observadores não treinados. Pode-se inferir, então, que um programa incorporando instruções, reforçamento diferencial, ensaio do compor- 
tamento, feedback e elogios pode ser efetivo para treinar bom relacionamento e melhorar a qualidade da conversação em idosos institucionalizados com limitações físicas e isolamento social. Essa melhora poderia favorecer o aumento da conversação por telefone com amigos ou parentes; entretanto, os participantes relataram não terem feito ligações telefônicas para amigos ou familiares.

A generalização de comportamentos sociais adequados por meio de treinos em ambientes em que os sujeitos normalmente interagem tem sido um tópico controverso na literatura.

Foxx, McMorrow, Bittle e Ness (1986) testaram a hipótese de que a generalização de habilidades sociais treinadas para um ambiente natural pode ser demorada para pessoas com longa história de comportamento social inadequado. A interação comportamental de dois grupos de residentes deficientes mentais idosos de uma comunidade foi medida em duas situações de generalização. As medidas foram realizadas antes, durante e depois que um dos grupos recebia treinamento de habilidades sociais por meio de um jogo. $\mathrm{O}$ jogo utilizava um conjunto especial de cartas que apresentava 48 situações sociais no tabuleiro de um jogo. Cada participante recebia um conjunto de 12 cartas com situações diferentes em cada jogo. Durante a intervenção, os participantes moviam seus jogadores no tabuleiro somente quando suas respostas eram apropriadas. $\mathrm{O}$ experimentador fornecia feedback positivo ou corretivo após cada resposta e modelava respostas apropriadas. O grupo controle simplesmente jogava sem ênfase ou interação comportamental. Os resultados mostraram que houve um aumento no número de interações apropriadas durante a intervenção e sugerem que a generalização para situações naturais de interação pode não ocorrer imediatamente após o treinamento e parece mais provável de ser observada em determinadas situações (com parceiros treinados) que em outras (na presença de parceiros não treinados).

Outro fator que pode causar o isolamento social são as vocalizações desconexas, exibidas, geralmente, por pacientes com demência.

Buchanan e Fisher (2002) pretendiam investigar a eficácia de uma avaliação funcional para direcionar o desenvolvimento de um tratamento com pacientes idosos com demência e vocalizações desconexas, e avaliar o tratamento com reforçamento não contingente (NCR). Vocalizações desconexas foram definidas como qualquer vocalização que pudesse ser ouvida a pelo menos 7,6 metros de distância. Reforçamento não contingente envolveu apresentar um estímulo com propriedades reforçadoras após determinados intervalos de tempo predeterminados e que não eram necessariamente contingentes a um determinado comportamento. A análise funcional implicava aumentos e reduções de estimulações sensoriais com duração de 15 segundos, tais como televisão ou música, que eram apresentadas (ou removidas) de forma contingente à ocorrência do comportamento-alvo. Participaram do estudo uma mulher de 89 anos, com demência e agitação, e um homem de 82 anos, com Alzheimer e demência vascular. Todas as sessões foram conduzidas em ambiente natural, aproximadamente no mesmo horário que as vocalizações desconexas costumavam ocorrer. Sessões com duração de 30 a 50 minutos foram conduzidas a cada dia. Estimulações 
em forma de ruído (por exemplo, pessoas conversando) foram apresentadas durante toda a linha de base e sessões do tratamento. Durante a linha de base, os dados do comportamento-alvo foram coletados sob as circunstâncias típicas particulares de cada residente. Nenhum reforçador foi entregue, e as enfermeiras foram instruídas a não interagir com o residente. Segundo os dados da análise funcional, cada ocorrência das vocalizações desconexas era seguida por atenção e estimulação sensorial em forma de música para a mulher, e por atenção para o homem. Isso pode significar que, quando os participantes ficavam algum tempo privados do acesso aos reforçadores, eles emitiam as vocalizações desconexas. Durante a condição de NCR, esses reforçadores foram apresentados em um esquema de razão fixa e não eram necessariamente contingentes ao comportamento-alvo, diminuindo a privação aos reforçadores. Os resultados indicaram que as vocalizações disruptivas diminuíram durante a condição de tratamento. Pode-se inferir, então, que o uso de análises funcionais e NCR pode ser útil no tratamento de vocalizações disruptivas em idosos com demência. Além disso, o tratamento resultou no maior acesso aos reforçadores funcionalmente relacionados ao comportamento-alvo, um importante dado para as instituições de cuidado.

\section{Atividades de vida diária}

É fato corriqueiro encontrar em residentes de asilos e clínicas geriátricas indivíduos com deficits de comportamento ou comportamento inapropriado referentes ao repertório comportamental exigido pelas atividades de vida diária. Os deficits dizem respeito, principalmente, à ociosidade, pois esses residen- tes tendem a permanecer em seus quartos e não participam das atividades disponíveis, e ao uso de toalete, devido à incontinência urinária, para a qual a conduta comum tem sido o uso de fralda geriátrica para evitar constrangimentos para o idoso. Essa conduta, embora prática para o cuidador, elimina qualquer possibilidade de modificação do referido comportamento. Os comportamentos inapropriados referem-se, principalmente, a comportamentos de vaguear, que pode ser entendido como entrar em ambientes restritos ou sair da instituição sem permissão.

Quattrochi-Tubin e Jason (1980) investigaram a efetividade de um procedimento de mudança ambiental para aumentar a participação de idosos em uma atividade, a interação entre os idosos e a quantidade de solicitações de ajuda. Participaram do experimento 20 homens e 79 mulheres com idades entre 55 e 97 anos, residentes em uma casa de repouso. Durante a coleta de dados, foram feitos dois tipos de observação: a) do contexto social da sala de estar: quantos residentes estavam presentes, quantos assistiam à televisão e interagiam entre si ou com a equipe; e b) das atividades: nível de participação voluntária dos residentes nas atividades disponíveis na sala de estar. Ao final da sessão de atividades, os residentes eram classificados em: participou ativamente, participou marginalmente ou não participou. Foi feito um delineamento experimental do tipo $\mathrm{ABAB}$, também chamado de delineamento de reversão. $\mathrm{Na}$ fase $\mathrm{A}$ (linha de base), as atividades normais dos residentes eram apenas observadas na sala de estar e na sessão de atividades. Na fase B (intervenção), um anúncio público avisava que café e biscoitos estariam disponíveis aos residentes na sala de estar. Os 
resultados revelaram que, na primeira introdução da intervenção, a média de residentes presentes, de residentes ativos e marginais aumentou em relação a primeira linha de base. $\mathrm{Na}$ segunda linha de base, a presença e a participação diminuíram, mas ainda foram maiores do que na primeira linha de base. A sessão de "café e biscoitos" serviu como um catalisador para as interações sociais. Muitos residentes relataram que, apesar de não tomarem café ou comerem biscoitos, gostavam de estar presentes na sala de estar durante as sessões de atividade e de lanche. A principal contribuição desse estudo foi a aplicação de uma técnica de controle de estímulos bastante simples que resultou no aumento de presença, participação e interação social em uma clínica geriátrica.

Gallagher e Keenan (2000) delinearam um programa para que os residentes se engajassem independentemente em atividades de lazer quando eles desejassem. Participaram 19 homens e 16 mulheres com idades entre 46 e 97 anos. Os materiais utilizados foram dardos, bingo, quebra-cabeças, livros, boliche e outros. Quando um residente era observado usando um desses materiais, era então anotado. $\mathrm{O}$ uso independente foi definido como o uso apropriado dos materiais por um ou mais residentes. Primeiramente, foram coletados dados durante duas semanas para avaliar a frequência do uso independente e espontâneo dos materiais sem nenhuma mudança ambiental. Então, foi utilizado o seguinte delineamento de tratamentos alternados: Lembrete Verbal (durante a janta, por uma semana, foi dado o seguinte aviso "Se alguém quiser fazer alguma atividade, fique a vontade em qualquer momento para realizá-la. Basta pegar o material e dar seu nome ao enfermeiro"); Lembrete em Cartaz (uma lista foi colocada com as várias atividades, durante duas semanas, juntamente com o lembrete verbal); Gerenciamento de Fichas (cada vez que o residente quisesse usar um material, daria seu nome e retiraria uma ficha para posterior troca por um prêmio; essa condição durou seis semanas); Preferência (semelhante à condição anterior; no entanto, o residente poderia escolher o prêmio; durou três semanas); Loteria (semelhante à condição anterior; no entanto, o reforço era em dinheiro; durou duas semanas); Manutenção (realizado durante seis semanas para avaliar se a atividade se mantinha na ausência da loteria em dinheiro).

Nas condições de lembrete verbal e em cartaz, não houve mudança nas frequências de uso de materiais. Isso pode ter ocorrido devido aos efeitos do programa não contingente de atividades, ou seja, os residentes poderiam estar satisfeitos com as atividades arranjadas pela equipe de funcionários. Nas condições de gerenciamento de fichas e itens de preferência, houve um pequeno aumento no uso dos materiais.

A condição de loteria produziu um aumento substancial no uso independente de materiais. $\mathrm{O}$ retorno à linha de base resultou em uma diminuição na frequência do uso independente dos materiais, embora não nos níveis observados inicialmente. Com a reintrodução da condição de loteria, as frequências elevaram-se novamente e se repetiram na fase de manutenção. Isso pode ter ocorrido devido às propriedades reforçadoras das próprias atividades, sugerindo que, uma vez que os residentes já haviam tido contato com as atividades, reforços elaborados não seriam mais necessários por muito tempo. 
Essa vida tipicamente sedentária de idosos residentes em instituições também foi relatada por Burgio et al. (1994). Os autores conduziram observações por 12 horas com 11 idosos de um asilo e notaram que os idosos permaneceram $87 \%$ desse tempo sem atividade. Com o objetivo de aumentar o engajamento de idosos com idades entre 85 e 95 anos e com demência em atividades da vida diária, Engelman et al. (1999) delinearam um estudo para avaliar o impacto de dicas e elogios verbais frequentes. A variável dependente primária foi o engajamento dos residentes, classificado em: a) engajamento apropriado (atividades para manutenção de independência, qualidade de vida, saúde mental ou física, como sentar-se à mesa, cozinhar, brincar com o cachorro, desenhar ou pintar); b) engajamento inapropriado (comportamento mal-adaptativo, como agressão, sair sem permissão, vocalizações inapropriadas); e c) falta de engajamento (quando o residente não participava de nem uma atividade, como sentar-se sozinho, dormir, ficar olhando para o nada). Durante a fase de intervenção, o assistente deveria usar as seguintes técnicas: a) fazer contato pessoal com cada residente a cada 15 minutos, pelo menos; b) fornecer elogio para os residentes por engajamentos apropriados; e c) oferecer escolha de pelo menos duas atividades, se o residente não estivesse engajado.

Os resultados demonstraram aumento consistente de engajamento para cada um dos residentes durante a intervenção. Esses resultados sugerem que o uso frequente de dicas e elogios verbais pelos assistentes aumentou a taxa de engajamento em idosos com demência. Após a introdução da intervenção, os residentes, que se engajavam em poucas atividades (conversar, comer, exercitar-se e ler), passaram a se engajar em outras atividades, como plantar flores, alimentar e cuidar de um passarinho e de um cachorro, sentar-se à mesa, jogar e fazer artesanato. $\mathrm{O}$ estudo de Engelman et al. sugere que o treinamento apropriado da equipe de cuidadores pode aumentar o engajamento de adultos com demência em atividades da vida diária.

Em uma série de estudos observacionais, Baltes, Orzech, Barton e Lago (1983) relataram que o caminhar e vários comportamentos de autocuidado raramente são seguidos de reforçamento pelos cuidadores dessas instituições. Mas, comportamentos dependentes, como solicitar por assistência, são prontamente reforçados. Os resultados de estudos de intervenção sugerem que técnicas de reforçamento podem ser efetivas para aumentar comportamentos apropriados e diminuir comportamentos inapropriados em pessoas idosas. Um exemplo é o estudo de Burgio, Burgio, Engel e Tice (1986), que avaliou o uso de um procedimento de dica e elogio para aumentar a distância das caminhadas e a independência de idosos dentro de uma instituição. $\mathrm{O}$ estudo foi desenvolvido com sete mulheres e um homem com idades entre 69 e 90 anos. As observações foram realizadas no refeitório e na sala de estar. Durante todas as fases do estudo, a observação no refeitório era iniciada quando era dada a oportunidade ao residente de andar até a mesa. Eram registrados o número de passadas realizadas e a duração do caminhar. A mobilidade e a interação social foram observadas por meio de amostragem de tempo na sala de estar durante o dia. Antes da intervenção, a mobilidade foi observada durante o almoço e o jantar no refeitório. Quando o residente 
entrava no refeitório, era solicitado que ele fosse até a mesa da maneira habitual. Se o paciente andasse, a distância e a duração eram anotadas. Nenhum elogio ou dica eram fornecidos. $\mathrm{Na}$ intervenção, se o residente não caminhasse até a mesa, uma dica verbal era fornecida pelo experimentador: "Seu médico decidiu que você precisa andar mais. Eu gostaria que você tentasse andar até a mesa hoje. Eu lhe darei toda ajuda de que você precisar." Elogios eram fornecidos pela caminhada, independentemente da velocidade, e pelo engajamento em conversações. No refeitório, dois sujeitos começaram caminhando a distância máxima durante a linha de base; os outros seis sujeitos mostraram um aumento considerável no caminhar durante a intervenção. Seis dos oito sujeitos também progrediram na deambulação mais independente. A generalização do caminhar foi observada para outras situações em quatro sujeitos. Os efeitos ainda foram observados após quatro meses.

Como citado anteriormente, outro problema comum entre os idosos é a incontinência urinária, que constitui um problema prevalente e sério entre pessoas idosas, principalmente entre os residentes de instituições de longa permanência.

Foxx e Azrin (1973) argumentam que, no caso de idosos sem limitações físicas, o objetivo de um programa de incontinência poderia ser traduzido em ensinar ao sujeito a autoiniciativa e a execução da sequência inteira de toalete independentemente. Quando há limitações físicas, o programa poderia ensinar o idoso a "solicitar" assistência de toalete à equipe quando a necessidade surgir. Tais programas envolvem não somente ensinar o idoso, mas também ensinar a equipe da ins- tituição a requerer tais comportamentos dos pacientes e executar o pedido quando feito.

Schnelle et al. (1983) comentam que o fenômeno de a equipe desempenhar as atividades de vida diária (alimentar, vestir, toalete) para/pelos idosos pode estar relacionado com a falta de conhecimento dessa equipe, considerando que, muitas vezes, é mais rápido desempenhar uma função para o idoso do que motivá-lo e esperar que ele complete a tarefa independentemente. Por isso, esses autores descreveram um sistema simples de gerenciamento comportamental para aumentar o uso apropriado de toalete em idosos institucionalizados.

Esse sistema, delineado para reduzir os problemas derivados da incontinência urinária, foi avaliado em duas instituições com um delineamento de grupo controle com pré e pós-teste. Os componentes primários do sistema foram dicas e contingência social de aprovação/desaprovação, que requereu, aproximadamente, 2,5 minutos por paciente por hora para administrar. Durante cinco dias, antes da intervenção, um ajudante apalpava as roupas e o corpo do idoso, uma vez a cada hora, entre 7 horas da manhã e 7 horas da noite, registrava "molhado" ou "seco" e se o paciente requeria ajuda para toalete. Os ajudantes foram instruídos a não conversar com os idosos durante a checagem, mas a dar ajuda de toalete se fosse solicitada. Após os cinco dias, esse procedimento foi mantido para o grupo controle três vezes por semana. Para o outro grupo, durante a intervenção, o ajudante checava cada um dos idosos toda hora para ver se estava molhado e fornecia uma dica, como, por exemplo, "Sr....., você necessita ir ao banheiro?”. A definição operacional de "solicitar ajuda para toalete" 
inclui resposta afirmativa à dica e independência para requerer a ajuda. Aprovação social era fornecida quando o idoso estava seco ou quando solicitava a ajuda para toalete, e consistiu em fornecer atenção e elogio (por exemplo, “Bom, você está seco. Não é mais confortável? Isso torna meu trabalho mais fácil e eu posso ter mais tempo para conversar com você") ou tocar gentilmente o idoso. Desaprovação social era fornecida quando o idoso estava molhado e consistiu em mostrar desapontamento ("Eu não entendo por que você se molha e não pede ajuda. Eu venho aqui toda hora"). Além disso, o ajudante limpava o idoso sem manter conversação e sem prestar serviço adicional. A frequência de uso correto de toalete aumentou para os sujeitos experimentais das duas instituições, mas não para o grupo controle. Os dados sugerem que a incontinência é mais um problema de gerenciamento da equipe do que problema de reaprendizagem, o qual pode ser remediado por procedimentos de gerenciamento com melhoria do tempo de trabalho com o idoso.

Uma das causas primárias de institucionalização de idosos é o comportamento de vaguear, por ser de dificil gerenciamento e consumir muito tempo de supervisão por parte do cuidador, em geral familiares (Feliciano, Vore, Leblanc, \& Baker, 2004; Heard \& Watson, 1999). Esse comportamento pode ser definido como o deslocamento sem meta, desorientado e contínuo, que ocorre quando o indivíduo move-se de uma área para outra sem necessidade aparente e sem planejamento prévio. Nesses casos, os funcionários precisam oferecer vigilância e monitoramento constante e, quando o comportamento de entrar em áreas restritas e perigosas ocorre, eles não podem ignorá-lo por completo.
Feliciano et al. (2004) realizaram um estudo com o objetivo de examinar o efeito do uso de uma barreira para diminuir a entrada em um escritório (área restrita), para uma mulher de 53 anos com desordem bipolar e provável demência. Antes da intervenção, um observador coletava os dados, divididos em períodos de cinco minutos, totalizando 1 hora e 30 minutos por dia. O dado era registrado quando a participante cruzava um tapete de 60 centímetros, localizado em frente à porta do escritório, que ficava aberta o tempo todo. Quando a participante entrava no escritório, o procedimento típico era um membro da equipe interagir, conversar, manter contato visual e redirecionar a participante para fora do escritório usando dicas físicas. A intervenção teve início com a colocação de uma barreira, que media $38 \mathrm{~cm} \times 104 \mathrm{~cm}$, com a mesma cor da porta, fixada à altura dos olhos, redirecionamento e reforçamento diferencial de outros comportamentos. Entrar resultava em imediato redirecionamento sem oferecer instrução verbal ou contato visual e com uso de dica física mínima. Foi utilizado o esvanecimento do estímulo, com a mudança da cor da barreira para branco e redução do seu tamanho para $25 \mathrm{~cm}$. Os resultados indicaram que o número de entradas inapropriadas teve uma redução de 95\% durante a fase de intervenção. A barreira visual e o redirecionamento diminuíram a quantidade de entradas, o que foi mantido por vários meses. A barreira pode ter levado a um aumento do custo da resposta para entrar no escritório, por requerer que a participante abaixasse para passar sob a barreira ou caminhasse através da barreira com ligeira resistência. A barreira foi utilizada como um estímulo discriminativo para diminuir a qualidade de atenção dos 
cuidadores e, apesar de aumentar o esforço da equipe para entrar no escritório, a taxa de aceitabilidade foi alta, talvez porque os efeitos foram altamente desejáveis e a equipe não foi solicitada a ignorar o comportamento da residente. A redução no tamanho da barreira alterou minimamente os resultados obtidos; entretanto, não foi investigado o efeito da remoção completa da barreira pela retirada gradual do estímulo.

Ainda sobre o comportamento de vaguear, Heard e Watson (1999) analisaram esse comportamento em quatro idosos com demência, residentes em uma instituição de longa permanência, por meio de observação direta. O comportamento de vaguear foi definido como passeios contínuos, desorientados e sem propósito que ocorriam quando o residente movia-se da sua área específica para outra dentro da estrutura física da instituição. Foram realizadas seis observações, por meio de um protocolo, para cada um dos participantes, para determinar a situação mais frequente associada com o vaguear. Os dados indicaram que as consequências mais prováveis que mantinham o comportamento eram a atenção, itens comestíveis e estimulação sensorial. Durante a fase de intervenção, essas consequências eram entregues se o comportamento não ocorresse em determinado intervalo e ficavam indisponíveis no caso de ocorrência do comportamento. Os resultados indicaram redução do vaguear durante a fase de intervenção, para todos os participantes, quando a consequência que mantinha esse comportamento era entregue contingente a outros comportamentos. A contribuição mais importante desse estudo foi a demonstração de que um comportamento, previamente atribuído a desordens neurológicas, pode ser sensível a consequências mediadas socialmente.

Os dois estudos anteriores apresentam soluções viáveis e de baixo custo, atuando sobre as condições ambientais para reduzir a ocorrência de comportamentos não desejados em idosos com comprometimento cognitivo e comportamental, quando o comportamento não pode ser ignorado por questões de segurança ou bem-estar dos residentes.

Stock e Milan (1993) examinaram os efeitos de três tipos de intervenções comportamentais usando dicas e reforçadores sobre mudança e seguimento de orientações nutricionais. Participaram do estudo 160 residentes de uma instituição para idosos com idade média de 86 anos. Três residentes serviram como participantes primários. Os outros residentes forneceram dados contínuos, mas não foram usados individualmente para uma análise suplementar dos efeitos do tratamento.

Um delineamento de reversão estendido no qual as condições eram apresentadas na sequência ABCDEDCA foi usado: A) linha de base (dicas: desenhos de corações eram utilizados para designar baixo colesterol e calorias modificadas nos quatro pratos de uma refeição); B) sem dicas (os desenhos de coração eram removidos); C) dicas (informação impressa, dicas verbais, menus e informação na hora da saúde), feedback (gráfico colocado em um quadro amarelo na entrada da sala de jantar) e reforçador social (contingente a relatos de escolhas saudáveis); D) dicas, feedback, reforçador social e loteria (cada vez que um residente escolhia um item de coração, ganhava uma ficha que poderia ser trocada por uma bola retirada de um vaso; se fosse uma bola premiada, ele escolhia um prêmio); E) dicas, feedback, reforçador social, loteria e 
aliança (era solicitado que os três participantes se aliassem no esforço de encorajar as práticas de boa alimentação).

Os três participantes primários demonstraram um aumento nas escolhas de itens alimentares saudáveis em resposta às dicas aprimoradas, feedback e reforçador social. Os outros residentes mostraram pouco aumento nessa condição. A adição da loteria resultou na maior porcentagem de escolhas saudáveis. Uma diferença importante entre os três participantes e os outros residentes foi que os participantes primários tinham sido informados da necessidade de modificar seus hábitos alimentares. A porcentagem de escolhas saudáveis retornou aos níveis iniciais quando as intervenções foram descontinuadas.

\section{Discussão}

A partir da presente revisão de literatura, a metodologia utilizada para avaliar as mudanças de desempenho de indivíduos idosos é fundamental para o desenvolvimento de estratégias de tratamento efetivas para essa população. As intervenções comportamentais nas áreas de interação social, independência na realização de tarefas simples e complexas, memória e tempo de reação, e deficits em comportamento verbal e social foram demonstradamente tão bem-sucedidas quando aplicadas ao indivíduo idoso quanto quando aplicadas a qualquer outra população. A conclusão mais importante deste trabalho é que o ambiente desempenha um papel fundamental não somente no desenvolvimento e na manutenção e comportamentos, mas também na prevenção do declínio comportamental no indivíduo idoso e que, portanto, tal declínio não deveria ser interpretado ex- clusivamente como um resultado inevitável da idade (Williamson \& Ascione, 1983).

Apesar do número relativamente pequeno de estudos que têm examinado o uso de técnicas comportamentais com idosos, esses procedimentos podem ser efetivos para melhorar aspectos tipicamente considerados como imutáveis na população idosa. Sendo assim, o conhecimento científico produzido na gerontologia comportamental ainda é incipiente, o que demanda a necessidade de pesquisas para analisar a incidência de deficits e excessos comportamentais na população idosa e nos ambientes institucionais, a natureza das interações da pessoa com o ambiente, a eficácia de procedimentos para autoadministração de comportamentos, treinamento e administração de cuidadores nas residências ou na comunidade (Burgio \& Burgio, 1986).

Uma das mudanças ambientais manipuladas por vários dos estudos analisados refere-se ao acesso a reforçadores. Lindsley (1964) propôs que muitas pessoas idosas são expostas a sistemas insuficientes de reforçamento. Os esquemas de reforçamento intermitentes, que controlam muitos dos desempenhos das pessoas jovens, podem ser inadequados para pessoas idosas (Baron \& Menich, 1985). Os idosos parecem responder melhor em ambientes que fornecem reforçamento contínuo, aplicados para acompanhar a taxa ou a frequência do comportamento emitido. Entretanto, os efeitos de esquemas de reforçamento nos idosos têm sido pouco examinados em laboratório ou em ambientes aplicados, e eles parecem ser uma área importante para pesquisas futuras (Burgio \& Burgio, 1986).

Alguns procedimentos também precisam ser adaptados. Tentativas de adaptar o procedimento de treino de toalete de Foxx e Azrin 
(1973) para o uso com idosos forneceram uma excelente lição. Originalmente desenvolvido para treinar o uso do toalete com deficientes, o procedimento de Foxx e Azrin é uma intervenção comportamental que inclui carga de fluido e correção. Relatos anedóticos de pesquisadores que utilizaram esse procedimento sugerem que a carga de fluido é contraprodutiva com idosos, cujas bexigas não acomodam grandes volumes de líquido (Burgio \& Burgio, 1986). Adicionalmente, os pesquisadores têm sido relutantes em utilizar procedimentos com correção com essa população pelas condições frágeis frequentemente apresentadas por esses indivíduos (Burgio \& Burgio, 1986). Consequentemente, os pesquisadores têm confiado no uso programado do banheiro e no reforçamento positivo para o tratamento desse tipo de problema (Schnelle et al., 1983).

Apesar da efetividade do tratamento, muitos procedimentos comportamentais permanecem sem uso por não serem aceitos pelos participantes, cuidadores e familiares (Kazdin, 1981). São necessárias pesquisas para avaliar a aceitabilidade e incentivar o uso de tratamentos comportamentais por cuidadores, médicos e enfermeiros que atuam em ambientes geriátricos (Burgio \& Burgio, 1986). Muitas pessoas idosas têm problemas significativos de saúde e requerem a presença constante de médicos e de enfermeiros. Sob essas circunstâncias, a coexistência de modelos de treinamento médico e comportamental é mais apropriada (Burgio \& Burgio, 1986). Então, se os analistas comportamentais quiserem aumentar sua participação no cuidado de idosos, é crucial que colaborem com médicos e enfermeiras. Outros aspectos retratados nos estudos apresentados são a importância e a necessidade de investimento na formação continuada dos profissionais que atuam em instituições que prestam serviços à população idosa, assim como a capacitação de cuidadores para que as pessoas em processo de envelhecimento sejam mantidas junto a seus familiares e incluídas na comunidade, gozando de independência e qualidade de vida.

Intervenções comportamentais em gerontologia têm produzido estudos inovadores e bem-executados (Adkins \& Mathews, 1999). Essas pesquisas têm demonstrado que mudanças positivas podem ser feitas na vida dos idosos. Permanece claro, no entanto, que uma perspectiva de solucionar problemas de idosos, sem o suporte do sistema social que desenvolve ou apoia seus comportamentos, não levará a uma mudança efetiva e que persistirá. A gerontologia comportamental pode mudar em uma direção madura se aderir, o máximo possível, às dimensões da análise do comportamento aplicada. Essa estratégia promoverá o crescimento, a efetividade e a humanização das intervenções comportamentais que melhoram a qualidade de vida dos idosos (Adkins \& Mathews, 1999).

\section{Conclusões}

Comparativamente aos esforços da área da psicologia como um todo, a análise do comportamento tem contribuído de forma ainda incipiente para a compreensão do processo de envelhecimento humano (Derenne \& Baron, 2002). Pelo senso comum, o envelhecimento tem sido visto mais como uma deterioração do sistema nervoso, que estaria imune às mudanças ambientais, e esta é, provavelmente, uma das razões pelas quais o tema não tem recebido maior atenção da parte dos analistas comportamentais. 
Outra possível razão é que a análise do comportamento ainda não cresceu suficientemente para abordar todas as questões de relevância comportamental. Derenne e Baron (2002), no entanto, sugerem ainda outra possibilidade, a de que a escassez de pesquisas de cunho analítico-comportamental sobre as variáveis que controlam o envelhecimento reflete um conflito que envolve as tradições de pesquisa na área de análise do comportamento.

Os métodos com base em estados estáveis de organismo único, descritos por Sidman (1960), constituem-se na estrutura principal das pesquisas na área comportamental. As características principais desses métodos podem ser sumarizadas como se segue: a) influências das variáveis independentes são avaliadas em função das alterações que elas produzem sobre o desempenho estável dos organismos individuais; b) o número relativamente pequeno de organismos estudados é compensado pela extensão e profundidade do exame de seus desempenhos; e c) o exame do desempenho individual sob diferentes condições controladas permite o estabelecimento de relações funcionais dentro de um mesmo indivíduo.

O poder desses métodos encontra-se amplamente documentado na literatura analítico-comportamental em termos das respostas fornecidas para uma ampla variedade de questões de interesse estritamente experimental/ conceitual ou aplicado. Mas, conforme destacam Derenne e Baron (2002), o estudo do envelhecimento levanta problemas específicos relacionados à aplicação desses métodos. Os principais aspectos das críticas dos referidos autores são apresentados em seguida.

Inicialmente, nos estudos sobre envelhecimento humano, os diferentes níveis de envelhecimento não podem ser impostos a um organismo único, pelo menos da mesma forma que a magnitude de um reforçador ou a concentração de uma droga. A idade comumente é tratada como uma variável do sujeito, da mesma forma que o são o gênero, nível econômico ou personalidade.

A ênfase da Análise do Comportamentalo é sobre o controle dessas variáveis por meio da escolha de organismos que dividem as mesmas características, o que apresenta a clara vantagem de reduzir as variações comportamentais que possam interferir com os efeitos das variáveis independentes, mas também a desvantagem de que o papel das variáveis do sujeito permanece obscuro.

Derenne e Baron (2002), assim como Baron e Perone (1998), defendem a noção de que o emprego exclusivo de organismos envelhecidos como sujeitos experimentais revela muito mais sobre as variáveis experimentais manipuladas do que possíveis influências do nível de envelhecimento dos sujeitos, e que, para se estabelecerem as influências do nível de desenvolvimento sobre um desempenho, é necessário que o nível de desenvolvimento de um organismo seja comparado com o de outro organismo em um nível diferente de desenvolvimento.

A definição de variáveis do sujeito ao invés da manipulação de variáveis independentes tem sido objeto de reserva dos analistas comportamentais. No entanto, alguns autores defendem a noção de que as abordagens consideradas tradicionais na psicologia não somente aceitam as variáveis de sujeito como legítimas, como também empregam delineamento de controle por grupos, e empregam estatística descritiva e inferencial. Sugerem, ainda, que diferenças na abordagem experi- 
mental isolaram a análise do comportamento da psicologia tradicional de outras disciplinas, tais como economia, ciências políticas e educação, e que se a análise do comportamento pretende fazer uma contribuição para a compreensão de processos socialmente importantes, tais como o envelhecimento humano, é preciso que haja uma reconciliação entre o seu método e os métodos mais convencionais (Derenne \& Baron, 2002; Baron, Perone, \& Galizio, 1991).

\section{REFERÊNCIAS}

Adkins, V. K., \& Mathews, M. (1999). Behavioral gerontology: state of the science. Journal of Clinical Gerontology, 5, 39-49.

Baltes, M. M., Orzech, M. J., Barton, E. M., \& Lago, D. (1983). The microecology of residents and staff: Bbehavioral mapping in a nursing home. Zeitschrift fur Gerontologie, 16, 18-26.

Baron, A., \& Menich, S. R. (1985). Reaction time of younger and older men: Effects of compound samples and prechoice signal on delayed matchingto-sample performances. Journal of the Experimental Analysis of Behavior, 44, 1-14.

Baron, A., Menich, S. R., \& Perone, M. (1983). Reaction time of younger and older men and temporal contingencies of reinforcement. Journal of the Experimental Analysis of Behavior, 40, 275-287.

Baron,A., \& Perone, M. (1998). Experimental design and analysis in the laboratory study of human operant behavior. In K. A. Lattal, \& M. Perone (Eds.). Hanbook of research methods in human operant behavior (pp. 45-91). New York: Plenum Press.

Baron, A., Perone, M., \& Galizio, M. (1991). Analyzing the reinforcement process at the human level: Can application and behavioristic interpretation replace laboratory research? The Behavior Analyst, 14, 95-105.
Baron, A., \& Surdy, T. M. (1990). Recognition memory in older adults: adjustment to changing contingencies. Journal of the Experimental Analysis of Behavior, 54, 201-212.

Birren, J. E., \& Schaie, K.W. (1996). Handbook of the psychology of aging. New York: Academic Press.

Brasil. (2003). Ministério da Saúde. Estatuto do idoso. Parecer n⿳0 1.301 .

Buchanan,J.A., \& Fisher,J.E. (2002). Noncontingent reinforcement as an intervention for disruptive vocalizations in Alzheimer's disease patients. Journal of Applied Behavior Analysis, 35, 99-103.

Burgio, L. D., \& Burgio, K. (1986). Behavioral gerontology: application of behavioral methods to the problems of older adults. Journal of Applied Behavior Analysis, 19, 357-366.

Burgio, L. D., Burgio, K. L., Engel, B. T., \& Tice, L. M. (1986). Increasing distance and independence of ambulation in elderly nursing home residents. Journal of Applied Behavior Analysis, 19, 357-366.

Burgio, L. D., McCormick, K. A., Scheve, A. S., Engel, B. T., Hawkins, A. et al. (1994). The effects of changing prompted voiding schedules in the treatment of urinary incontinence in nursing home residents. Journal of the American Geriatrics Society, 42, 315-320.

Carvalho Filho, E. T. (2000). Geriatria: fundamentos, clínica e terapêutica. São Paulo: Atheneu.

Catania, A. C. (1999). Aprendizagem: comportamento, linguagem e cognição. (4. ed.). (D. G. Souza et al., Trad.). Porto Alegre: Artes Médicas Sul.

Creutzberg, M., \& Santos, B. R. L. (2000). “...se a gente não tem família, não tem vida!" Concepções de famílias de classe popular cuidadoras da pessoa idosa fragilizada. Revista Gaúcha Enfermagem, 21, 101-112.

Derenne, A., \& Baron, A. (2002). Behavior Analysis and the study of human aging. The Behavior Analyst, 25(2), 151-160. 
Engelman, K. K., Altus, D. E., \& Mathews, R. M. (1999). Increasing engagement in daily activities by older adults with dementia. Journal of Applied Behavior Analysis, 32, 107-110.

Feliciano, L., Vore, J., Leblanc, L.A., \& Baker, J. C. (2004). Decreasing entry into a restricted area using a visual barrier. Journal of Applied Behavior Analysis, 37, 107-110.

Foxx, R. M., \& Azrin, N. H. (1973). Toilet training the retarded. Champaign, Ill.: Research Press.

Foxx, R. M., McMorrow, M. J., Bittle, R. G., \& Ness, J. (1986). An analysis of social skills generalization in two natural settings. Journal of Applied Behavior Analysis, 19, 299-305.

Gallagher, S. M., \& Keenan, M. (2000). Independent use of activity materials by the elderly in a residential setting. Journal of Applied Behavior Analysis, 33(3), 325-328.

Green, G. R., Linsk, N. L., \& Pinkston, E. M. (1986). Modification of verbal behavior of the mentally impaired elderly by their spouses. Journal of Applied Behavior Analysis, 19, 329-336.

Hall, R.V. (1974). Managing behavior - behavior modification: The measurement of behavior. Lawrence, Kansas: H \& H Enterprises.

Heard, K., \& Watson, T. S. (1999). Reducing wandering by persons with dementia using differential reinforcement. Journal of the Applied Behavior Analysis, 32, 381-384.

Henry, L. M., \& Horne, P.J. (2000). Partial remediation of speaker andlistener behaviors in people with severe dementia. Journal of Applied Behavior Analysis, 33, 631-634.

Kazdin, A. E. (1981). Acceptability of child treatment techniques of treatment efficacy and adverse side effects. Behavior Therapy, 12, 493-506.

Lindsley, O. R. (1964). Geriatric behavioral prosthetics. In R. Kastenbaum (Ed.). New thoughts in old age (pp. 41-61). New York: Springer.
Maria, A. A. R. (2003). Biografia do abandono. São Paulo: Colégio Pentágono.

Néri, A. L. (2001). Velhice e qualidade de vida na mulher. In A. L. Néri (Org.). Desenvolvimento e envelhecimento: perspectivas biológicas, psicológicas e sociológicas (pp. 161-200). Campinas, SP: Papirus.

Pinkston, E. M., \& Linsk, N. L. (1984). Care of the elderly: A family approach. New York: Pergamon.

Plaud, J. J., Gillund, B., \& Ferraro, F. R. (2000). Signal detection analysis of choice behavior and aging. Journal of Clinical Geropsychology, 6, 73-81.

Praderas, K., \& MacDonald, M. L. (1986). Telephone conversational skills training with socially isolated, impaired nursing home residents. Journal of Applied Behavior Analysis, 19, 337-348.

Quattrochi-Tubin, S., \& Jason, L. S. (1980). Enhancing social interactions and activity among elderly through stimulus control. Journal of Applied Behavior Analysis, 13, 159-163.

Schnelle, J. F., Traughber, B., Morgan, D. B., Embry, J. E., Binion, A. F., \& Coleman, A. (1983). Management of geriatric incontinence in nursing homes. Journal of Applied Behavior Analysis, 16, 235-241.

Sidman, M. (1960). Tactics of scientific research. New York: Basic Books.

Skinner, B. F. (1983). Intellectual self-management in old age. American Psychology, 38, 239-244.

Stock, L. Z., \& Milan, M. A. (1993). Improving dietary practices of elderly individuals: The power of prompting, feedback, and social reinforcement. Journal of Applied Behavior. Analysis, 26, 379-387.

Williamson, P. N., \& Ascione, F. R. (1983). Behavioral treatment of the elderly: Implications for theory and therapy. Behavior Modification, 7(4), 583-610.

Wisocki, P. A. (1991). Behavioral gerontology. In P. A. Wisocki (Ed.). Handbook of clinical behavioral therapy with the elderly client (pp. 3-51). New York: Plenum Press. 